\title{
Designing for Diabetes Decision Support Systems with Fluid Contextual Reasoning
}

\author{
Dmitri S. Katz \\ The Open University \\ Milton Keynes, UK \\ dmitri.katz@open.ac.uk
}

\author{
Blaine A. Price \\ The Open University \\ Milton Keynes, UK \\ b.a.price@open.ac.uk
}

\author{
Simon Holland \\ The Open University \\ Milton Keynes, UK \\ simon.holland@open.ac.uk
}

\author{
Nicholas Sheep Dalton \\ Northumbria University \\ Newcastle upon Tyne, UK \\ nick.dalton@northumbria.ac.uk
}

\begin{abstract}
Type 1 diabetes is a potentially life-threatening chronic condition that requires frequent interactions with diverse data to inform treatment decisions. While mobile technologies such as blood glucose meters have long been an essential part of this process, designing interfaces that explicitly support decision-making remains challenging. Dual-process models are a common approach to understanding such cognitive tasks. However, evidence from the first of two studies we present suggests that in demanding and complex situations, some individuals approach disease management in distinctive ways that do not seem to fit well within existing models. This finding motivated, and helped frame our second study, a survey $(n=192)$ to investigate these behaviors in more detail. On the basis of the resulting analysis, we posit Fluid Contextual Reasoning to explain how some people with diabetes respond to particular situations, and discuss how an extended framework might help inform the design of user interfaces for diabetes management.
\end{abstract}

\section{AUTHOR KEYWORDS}

Health; chronic conditions; mHealth; apps; pervasive computing; ubiquitous computing; wearable interaction; quantified self; personal informatics; Internet of Things; digital health.

\section{ACM Classification Keywords}

H.5.m. Information interfaces and presentation (e.g., HCI): Miscellaneous.

\section{INTRODUCTION}

Common chronic conditions such as obesity, diabetes, asthma, and hypertension have both widespread quality of life implications and are also a burden on healthcare systems worldwide. Informed self-management is essential in these conditions for improving outcomes. Consequently, this attracts significant research interest in many fields, including Human Computer Interaction. Mobile computing has the potential for low-cost scalable approaches to support better health decisions and behaviors, for example by delivering the right message, in the right way, at the right

This work is licensed under a Creative Commons Attribution International 4.0 License.

CHI 2018, April 21-26, 2018, Montreal, QC, Canada (C) 2018 Copyright is held by the owner/author(s). ACM ISBN 978-1-4503-5620-6/18/04. https://doi.org/10.1145/3173574.3174199 time [10]. A smart device could potentially become an automated 'doctor in the pocket' health system, intervening, guiding, and altering lifestyle and medical choices. However, designing and implementing effective behavior change interventions remain challenging [8], while creating and promoting Decision Support Systems (DSS) raises critical ethical questions. Meredith and Arnott [16] outlined key concerns: who bears responsibility for recommendations; potential impact on the user's cognitive structures; and dangers to autonomy such as interfering with the user's right to make final choices. Stawarz et al. [22] concur, noting how automated reminder systems can inhibit independent habit formation, thereby causing vital behaviors such as medication adherence to become technology dependent. One potential approach to alleviate such concerns could be to support rather than replace existing self-care models and behaviors [19]. For this and other reasons, it is essential for designers to understand the existing practices and mental processes of those who live with these chronic conditions.

\section{Diabetes}

Diabetes is a serious and prevalent condition with current estimates at over 400 million patients worldwide [1]. Type 1 Diabetes (T1D), which afflicts $5-10 \%$ of those with diabetes, is an autoimmune disease that destroys the body's ability to produce the hormone insulin. People with T1D must either wear an insulin pump, which continuously infuses insulin, or take multiple daily injections of insulin to control Blood Glucose (BG) levels. Those who are on multiple dose insulin therapy commonly take both slow acting insulin (basal) to meet persistent requirements, and a fast acting insulin (bolus) either for meals or to reduce excessive BG levels (hyperglycemia). Commonly accepted BG levels are in $70-140 \mathrm{mg} / \mathrm{dl}$ ranges. Excessively high BG must be treated with insulin to avoid diabetic coma, while excessive low BG (hypoglycemia) must be treated, for example with oral glucose, to avoid seizures. While T1D can be successfully managed with a carefully controlled lifestyle and the correct use of medications, diabetes management remains challenging. This is due in part to the complexity of care caused by the great number of hard to control factors that can cause BG fluctuations, such as stress, sickness, and variable insulin sensitivity. A recent study found a majority of individuals in treatment do not achieve recommended guidelines [18]. Although some aspects of diabetes management are disease specific, others, such as healthy diet, sufficient exercise, coping with stress, proper use of medications, and 
monitoring [2] are not, suggesting the potential wider relevancy of the research described.

\section{Supporting technologies}

With increased adoption of smartphones, diabetes apps have become a popular approach to supporting situated daily management. They have largely focused on offering tools to collect and reflect on diverse personal biometrics and contextual factors. However, these systems are still largely dependent on manual entry to acquire such data, which can be a significant barrier to adoption [3]. In addition, some research has suggested that many visual paradigms used in order to make sense of this data are too cognitively demanding for situated use [11]. Despite these issues specifically with apps, daily interaction with digital devices is a key component of T1D management, and has been since the late 1980's, with the advent of portable BG meters. These meters allow convenient measurement of BG values from a small drop of blood taken from the fingertip, enabling patients to self-monitor and flexibly adjust treatment decisions. Such real-time information facilitates tighter diabetes control, which can increase quality of life and reduce long-term complications. However, even with 4-8 tests per day, such measurement allows only 'snapshots' of glycemic values, leaving what happens between tests, along with rate and direction of change, unknown. The Continual Glucose Monitor (CGM) allows BG values to be automatically sampled many times per hour, and seen at will on a glanceable display, such as a proprietary device, smartwatch, or smartphone. This higher sample rate allows an arrow that predicts both rate and direction of change. A related technology is the Flash Glucose Meter (FGM), which is dependent on manual scanning for readings. While technically incorrect, we use the common term CGM to refer to both systems, due to UI similarities.

\section{Self-management}

Modern T1D treatment requires: self-monitoring BG levels and lifestyle factors; analyzing this information; and dynamically adjusting relevant variables accordingly. Since medical staff cannot always be on hand, patients (or their caregivers) must take primary responsibility for daily treatment decisions [7]. Therefore, it is essential to develop systems for the user, and not just health care professionals.

\section{HCI AND COGNITIVE MODELS}

Theoretical considerations have served an important function in the design of health interventions [15] by supporting the transference of acquired knowledge between related domains [9]. This is reflected in HCI, where there have been numerous studies engaging with or inspired by behavior theory. Examples include [13] which incorporated multiple elements of behavior theory literature and [6] which investigated goal-setting theory in a persuasive technology context. Notably, the behavior change wheel [17], sought to bring together all the potential tools for developing effective behavior change interventions. While behavioral [17] or stage-based models [5] might potentially play an important role in designing interventions for supporting diabe- tes self-management, in this paper we will specifically focus on cognitive models, as they are more directly related to our primary concern in this research: the mental processes with which people interact with information to make decisions [15]. The following sections review select cognitive theories relevant to such processes.

\section{Dual-process cognitive theories}

Dual-process cognitive theories are a leading conceptual approach to understanding the reasoning process [23]. Such cognitive theories exist in numerous permutations, with diverse empirical and theoretical backing [6]. Evans and Stanovich [6] define a generalized version of dual-process theory as:

“...one in which rapid autonomous processes (Type 1) are assumed to yield default responses unless intervened on by distinctive higher order reasoning processes (Type 2). What defines the difference is that Type 2 processing supports hypothetical thinking and loads heavily on working memory."

Such dual-process approaches have been influential in HCI. $\mathrm{Li}$ et al. [12] researched reflection on personal data to increase self-knowledge, popularly known as the Quantified Self (QS), and observed two primary phases of interaction: maintenance and discovery. In the former, participants were primarily trying to sustain the behaviors or steps needed to achieve established goals. This phase, with its low cognitive demands is analogous to Type 1. In the latter phase, people were asking questions, trying to establish cause-and-effect relationships and goals, analogous to Type 2. Mamykina et al. [14] researched how specifically supporting reflective processes can lead to realization of cause and effect relationships, thereby enabling diabetes self-management behaviors. This research led to the Sensemaking theoretical framework [15], which identifies the cognitive processes used in self-management behaviors, and is essentially a dual-process theory. This variant, especially relevant to this paper, proposes a dynamic interaction between two modes of daily management, habitual and sensemaking. Further, it presents three key stages of decision-making that occur in both modes: perceiving new information related to condition, understanding this information, and action based upon this information. Sense-making behaviors are typically triggered when the individual notes a 'gap', such as an unexplained out of range BG level. In such instances the new information does not fit into an established self-care heuristic, and the individual must experiment with new behaviors (sensemaking). Such hypothesis testing leads to the formation of new models which eventually can be used in an effortless and largely unconscious manner. This theory emphasizes that the ability to operate predominantly in the habitual mode is important for sustainable self-care, as sensemaking is cognitively demanding.

\section{Tri-process theories}

While there are many examples of dual-process theories in the literature [6], there are far fewer tri-process theories. Stanovich [21] suggested that it might be useful to distin- 
guish between two aspects of Type 2 thinking: reflective and algorithmic thinking. Alternatively, Varga and Hamburger [23] proposed a tri-axial continuous model of control, effort, and speed, asserting that this model allows for better classification of real world behaviors: the skilled automobile driver on an unfamiliar road offers a clear example. Such a driver has an acquired set of driving skills, which allow for fluid control over the vehicle in most circumstances. But, in a context of unfamiliar roadways, she must remain attentive and actively engaged. The same basic skills are still applied, but the driver cannot function in the largely reflexive mode used on the habitual daily commute. This example offers intriguing parallels to the person with T1D who applies a set of acquired self-management models to navigate the shifting contexts of daily life.

This paper investigates how People with Type 1 Diabetes (PwT1D) make decisions for disease self-management both in routine and abnormal circumstances. Based on this research, we posit a cognitive process, under-explored in the literature, that allows people with diabetes to engage in rapid stream-of-consciousness navigation of complex situations. We present evidence for this process, which we refer to as Fluid Contextual Reasoning (FCR), and suggest that it can serve a practical function by allowing a workaround for the respective limitations of more typical 'fast' and 'slow' thinking. We conclude by showing how aspects of $F C R$ are already being supported by more recent technologies, and suggest ways in which, based on this expanded model, User Interfaces (UIs) for diabetes management could more closely address users' cognitive requirements within specific contexts. This research may have implications for scalable health interventions more generally, and may help embody an ethical path forward for supporting rather than supplanting functional self-care knowledge and models. Even more generally, this work, though domain specific, could have relevance to other domains that require informed but nonexpert users to interact frequently with vital complex data. In order to further our understanding of these lived practices of decision-making, we conducted the following studies.

\section{STUDY 1}

The first, study 1 , was a talk-aloud user-interaction study to investigate the effectiveness of diabetes apps in helping users to interact with data. In this section we give brief sample findings from the study, which while often supporting the Mamykina et al. [15] theory for diabetes selfmanagement, also suggested particular aspects of behavior not well accounted for by this framework. This motivated and helped frame Study 2, a survey study $(\mathrm{n}=192)$ to investigate those behaviors in more detail.

\section{Methods}

We initiated, recorded, transcribed, and analyzed mediated sessions in which 16 PwT1D talked aloud as they reflected on 14 days of pre-collected diabetes relevant data (BG, carbohydrates, exercise, insulin dosages) within 6 popular diabetes logging apps on an iPhone $5 \mathrm{~s}$. Third party data was used to facilitate comparability. Interviews were variable length semi-structured user interaction sessions lasting between 20-65 min. Participants were recruited through the Berlin Diabetes and Technology Meetup, snowball, and convenience sampling. Interviews were conducted in 2015 and 2016. The inclusion criteria were T1D, over age 18, and speaking conversational English. Age range was from $25-49$ years with a mean age of 34 years. Time since diagnosis ranged from 2-31 years, with a mean of 14. Gender was 5 female and 11 male. University Ethics Committee granted approval. No financial incentives were offered.

\section{Findings}

The following section reports on participants' responses during the user-interaction sessions. We use the [15] sensemaking framework as a filter through which to analyze these selected excerpts.

\section{Support for a dual-process Sensemaking perspective}

During the user-interaction sessions, many participants provided detailed descriptions of the steps they undertook to successfully cope with diabetes. P11 for example said: "I think decision-making (on) diabetes...it has a lot of steps. The first step is just recognizing you have a problem ... like I have a lot of highs. The second step would be...when do I have a lot of highs? What did I do? Did I do certain things and then had a lot of highs? What...could the problem be? And then take action and then play around a little bit... but carefully." In this quote we see evidence of the multiple stages described in [15], the first being the perception of a gap, ("you have a problem"). P11 then describes the inference stage of considering the circumstances, leading to the construction of a hypothesis, which is then followed by experimentation. P11 continues, "And then in the last step would be ... when I recognize that I had this problem in the morning, first I was trying a little bit with the basal dosage and then it helped a little bit and then I thought okay now I can play a little more extreme. So I changed my basal dosage from 12 to 19. OK that's kind of extreme but it worked out fine. My blood sugar is good again." P11 has detailed the hypothesis testing, leading to the discarding of the old model (I need $x$ units of slow acting insulin at night) to be replaced by the new model. Now that this new model for insulin dosages has been validated, P11 can return to the habitual behavior of a set nightly insulin dosage. To review, in the preceding example we can see the full cycle of the described framework. The 'gap' is the recognition that there are too many elevated BG levels, which initiates a series of inferences, which lead to experimentations, and finally the restoration of a habitual behavior. As long as BG values are judged to be satisfactory, a standardized treatment has been decided upon, no longer requiring cognitive effort.

A more routine use of sensemaking can be seen in P7 looking through several days data in a digital logbook: “... they've taken the same amount of insulin with way less carbs in the morning and they still had problems keeping the blood sugar down. So... they need to ... in the morning... increase the amount of insulin that it normally take 
for a meal." In this case the gap is once again elevated morning BG, the hypothesis is insufficient morning insulin, a second case is observed in the logbook with less carbohydrate intake, which further supports this hypothesis. Presumably this would then be tested to see if it improves after breakfast BG levels, without undue hypoglycemia.

However, we also found examples that did not fit as easily in the described schema. P4 describes a process of managing diabetes during a Saturday night of partying. P4 upon reflecting on recorded data showing non-stable BG values on a Saturday night relates, "... at night to have a blood sugar like this is always difficult because when you ... drink then you have a problem to get out of there [unwanted BG level] depending on how much alcohol your body is having. ... the most dangerous thing is drinking sweet stuff ... also I know that and at 3:00 in the night 55 [hypoglycemia] so I mean when you drink no sweet stuff then you'd just like Schnapps [an unsweetened concentrated alcohol]... then it's not a big issue when the only issue is that getting in a low sugar because then your body can't take sugar again and it's busy with alcohol when you drink sweet stuff and beer and this stuff then you need almost to inject because your blood Sugar's going up but this is not allowed at all to inject for alcohol so I think it's really good to drink schnapps and dance ... and drink cola because the way how to you can resolve the night because you can control your blood sugar with dancing, smoking weed, and pushing it up with Coca-Cola again" In this quote we see P4 balancing multiple factors that can have an unpredictable effect on BG levels, using multiple mechanisms to cope with potentially risky behaviors. We see habitual fixed models, such as to not inject insulin while drinking alcohol so as avoid the risk of severe hypoglycemia, and to not drink sweetened alcohol, as that will overly raise BG levels. However there are also context dependent models, such as using dancing to lower BG levels while drinking (how much? how vigorous?), or to use Coca-Cola to raise BG levels. It is not clear from the quote which effect marijuana has on BG levels, but either for medical utility or quality of life, it has been incorporated into P4's dynamic equation. With so many unpredictable factors on BG levels, as well as potential risks, it would be difficult to classify this process entirely as an 'effortless' habitual mode, but with all the cognitive impairment, distractions, and frequent in the moment adjustments, enacting such a process of diabetes management would be difficult to classify this in a strict sensemaking mode. This appears to have some similarities to the aforementioned expert driver, who can draw upon multiple skills in a fluid manner, adapting them to shifting contexts.

\section{Discussion}

As well as supporting the Mamykina model, we also describe a mode of operation that appears to allow those with sufficient accumulation of self-management models, to assess and act upon complex context-dependent multivariate data. Further, they can do this in a flowing and relatively low-cognitive manner that allows them to process infor- mation that is too complex and has too many variables and unknowns to be literally 'solved'. This appears to be achieved through linking and adjusting multiple co-existing models. Further differentiating this mode from sensemaking, we do not see the recognition of a clearly defined 'gap', effortful thinking, nor necessarily a process of learning leading to the creation of new models nor discarding of old ones. We have labeled this mode of thinking fluid contextual reasoning (FCR). FCR draws on behaviors at the intersection of the dual-processes (Fig. 1); however, it combines them in new and distinctive ways - as discussed below. Table 1 provides a table (non-exhaustive) that suggests potential categorization.

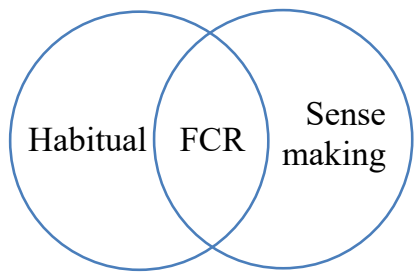

Figure 1 Self-Management modes

\begin{tabular}{|c|c|c|}
\hline $\begin{array}{l}\text { Habitual } \\
\text { (Type 1) }\end{array}$ & FCR & $\begin{array}{c}\text { Sensemaking } \\
\text { (Type 2) }\end{array}$ \\
\hline $\begin{array}{l}\text { Implicit, } \\
\text { Effortless }\end{array}$ & $\begin{array}{c}\text { Low-demand Active } \\
\text { Engagement }\end{array}$ & $\begin{array}{l}\text { Explicit, } \\
\text { Effortful }\end{array}$ \\
\hline $\begin{array}{l}\text { Applying existing } \\
\text { static, rule-based } \\
\text { models }\end{array}$ & $\begin{array}{c}\text { Adapting/combining } \\
\text { multiple existing } \\
\text { models }\end{array}$ & $\begin{array}{l}\text { Hypothesis testing/ } \\
\text { Creating new models }\end{array}$ \\
\hline $\begin{array}{l}\text { Algorithms: Prac- } \\
\text { ticed / discrete }\end{array}$ & $\begin{array}{l}\text { Algorithms: ap- } \\
\text { proximate }\end{array}$ & $\begin{array}{l}\text { Algorithms: demand- } \\
\text { ing or novel }\end{array}$ \\
\hline $3 * 3=9$ & $\begin{array}{l}3.1 * 2.9 \approx \\
3 * 3=9\end{array}$ & $3.1 * 2.9=8.99$ \\
\hline $\begin{array}{l}\text { Reflexive/ } \\
\text { Reacting }\end{array}$ & $\begin{array}{l}\text { Improvisation/ } \\
\text { Navigating }\end{array}$ & $\begin{array}{l}\text { Reflection/ } \\
\text { Solving }\end{array}$ \\
\hline Chunked memory & $\begin{array}{c}\text { Connecting Chunked } \\
\text { memory }\end{array}$ & $\begin{array}{l}\text { Loading working } \\
\text { memory }\end{array}$ \\
\hline "If this, then that" & $\begin{array}{l}\text { "Some combination } \\
\text { of this and that" }\end{array}$ & $\begin{array}{l}\text { "Could this cause } \\
\text { that?" }\end{array}$ \\
\hline
\end{tabular}

Table 1 FCR proposed properties

\section{Habitual}

We define this mode as reflexive responses, habitual behaviors, or scripts activated in specific situations. Accessing such models allows for the low cognitive demands needed for rapid responses. These can be either a single action or a sequence of previously learned steps, models, or formulas. A multi-part habitual model might be: for elevated BG take a correction shot of 1 unit insulin per $50 \mathrm{mg} / \mathrm{dl}$, drink water, test again in 1 hour. As this response is already learned and tested, there is no need to contextually re-adjust.

\section{Fluid Contextual Reasoning}

We propose FCR is a combination of models being adjusted and applied to a specific context with increased awareness of how factors relate to past, present, and future to allow 'navigation' through a time continuum. An example might 
be rather than just counting carbohydrate content of a meal to calculate insulin dosages, considering other factors such as exercise that might occur hours later, recent trends in insulin sensitivity, and amount of fat in meal. Indicative of this state is stream of conscious layering and combining of models, with little demand on working memory, and little concern for establishing new self-care models.

\section{Sensemaking}

A recognition of an unexplained event (gap) leading to: discovery of a new pattern; cause and effect analysis; explanation of what caused an event to occur; or a learning process, with conscious creation or revision of specific model(s). An example might be: Through experience one realizes that eating pizza typically causes hypoglycemia, followed by hyperglycemia. One hypothesizes this could be due to combination of considerable carbohydrate and fat which delays digestion. Therefore, it makes sense to try splitting insulin bolus dosages next time to see if that helps. If this is confirmed, this can transition to a habitual model.

\section{STUDY 2}

In order to further investigate the FCR hybrid-mode hypothesis, we conducted a survey inquiring about the decision-making processes of PwT1D in relation to diabetes self-management in specific circumstances. We found evidence for widespread use of FCR as defined in the previous section, across all demographics, particularly in association with of CGM adoption and increased number of insulin boluses. In the following sections we will review the methods, procedure, and findings.

\section{Methods}

We created a survey developed through an iterative process with PwT1D with the initial questions being discussed and repeatedly revised. This was followed by a prototype survey being taken by three participants online, with further revisions based on answers and feedback after each session. The survey was then finalized and submitted for ethics approval. During our earlier research, we had found participant recruitment challenging, therefore we decided to use an online survey to increase sample size. Inclusion criteria were $\mathrm{T} 1 \mathrm{D}$, in medical treatment, and a minimum of 18 years of age. The survey was distributed online through social media including multiple T1 diabetes Facebook groups, Twitter, and, after undergoing an approval process, the online portals for the Diabetes UK and US based TU Diabetes online community. There were no incentives offered other than the appeal to help with diabetes research and the option to receive outcomes.

\section{Survey contents}

The first part of the survey was demographic in nature, establishing age, gender, duration of illness, diabetes devices used, and frequency of BG testing and insulin injections. This was then followed by a series of open-ended questions, allowing for more in depth information.

\section{Procedure}

Data were exported from a Google form, imported into an Excel spreadsheet, and then anonymized. Of the $n=206$ initially collected surveys, 14 were eliminated ( 3 completed under observation during the development process, 4 duplicates, 1 that was underage, and 6 that were judged too incomplete to provide meaningful data), for a total of $n=192$ responses.

\section{Coding}

The extended framework (see Table 1) was used as a schema, which was expanded by domain specific self-care models extracted from the collected responses. Such categories included: insulin, exercise, diet, blood glucose, testing, context, devices, time, place, health cycles, interfaces, etc. After the lead author composed the initial coding schema, it was applied to the 20 responses (Inter-rater reliability Cohens Kappa $=0.698$ ). A secondary author then reviewed the responses, and the coding schema was discussed and refined. The lead author, with frequent discussions, cocoding, and comparisons with secondary authors then coded all data. For example, the habitual mode category for insulin use included: fixed insulin/carb ratios, fixed BG correction factors, and fixed insulin doses. The FCR category for insulin use included: taking into account factors such as insulin on board (when not monitored by device), insulin sensitivity in relation to a specific context, intentionally waiting variable times between bolus and meal, microdosing insulin based on context, adjusting dosages according to trends. Sensemaking activities including: building self-management models, researching how context changes insulin sensitivity, retrospective analysis of insulin effect, calculating new insulin/carb factors, and experiments to determine new fixed dosages. After all responses had been classified as habitual, FCR, sensemaking, or nonclassifiable, counts were summed, and analyzed.

\section{Findings}

The following sections review the frequency of answers classified as either habitual, FCR, or sensemaking from the survey. Table 2 reviews this information sorted by demographic, while Table 3 reviews the responses to the openended questions. The " $n$ " in the table refers to total number of classified respondents within a specific demographic; this number can be lower than total participants due to answers that were missing, vague, or non-relevant. Totals show the count of participants who have been classified as exhibiting at least one instance of the three behaviors, emphasizing individual capability for exhibiting a mode of decision-making. For example, if P015 were to be classified with 5 instances of Habitual, 0 instances of FCR, and 6 instances of Sensemaking, this would be indicated as $(1,0,1)$.

\section{Cognitive mode in relation to demographics}

In this section, we review the three modes in relation to demographic information. We found that while habitual and sensemaking were most frequent, all three states were prevalent across all demographics. FCR appeared to be significantly associated with CGM usage, might have a slight in- 
crease with the first 10 years since diagnosis, and a small but significant correlation with bolus and testing frequencies.

\begin{tabular}{|c|c|c|c|c|c|c|c|}
\hline \multicolumn{8}{|c|}{ Number of participants who have exhibited a given behavior } \\
\hline & \multirow[b]{2}{*}{$\mathrm{n}=$} & \multicolumn{2}{|c|}{ Habitual } & \multicolumn{2}{|c|}{ FCR } & \multicolumn{2}{|c|}{ Sensemaking } \\
\hline & & $n>0$ & $\%>0$ & $n>0$ & $\%>0$ & $n>0$ & $\%>0$ \\
\hline \multicolumn{8}{|c|}{ Age in years } \\
\hline $18-24$ & 25 & 23 & 92 & 19 & 76 & 25 & 100 \\
\hline $25-34$ & 51 & 50 & 98 & 38 & 75 & 50 & 98 \\
\hline $35-44$ & 42 & 39 & 93 & 27 & 64 & 40 & 95 \\
\hline $45-54$ & 30 & 27 & 90 & 18 & 60 & 28 & 93 \\
\hline $55-64$ & 30 & 25 & 83 & 26 & 87 & 29 & 97 \\
\hline $65^{+}$ & 13 & 12 & 92 & 10 & 77 & 13 & 100 \\
\hline n.a. & 1 & 1 & 100 & 1 & 100 & 1 & 100 \\
\hline \multicolumn{8}{|l|}{ Gender } \\
\hline female & 146 & 135 & 92 & 105 & 72 & 143 & 98 \\
\hline male & 43 & 39 & 91 & 31 & 72 & 40 & 93 \\
\hline other & 3 & 3 & 100 & 3 & 100 & 3 & 100 \\
\hline \multicolumn{8}{|c|}{ Time since diagnosis in years } \\
\hline$<1$ & 7 & 6 & 86 & 4 & 57 & 7 & 100 \\
\hline $1-2$ & 14 & 14 & 100 & 9 & 64 & 13 & 93 \\
\hline $3-5$ & 15 & 12 & 80 & 11 & 73 & 15 & 100 \\
\hline $6-10$ & 14 & 14 & 100 & 11 & 79 & 14 & 100 \\
\hline $11-20$ & 43 & 41 & 95 & 33 & 77 & 42 & 98 \\
\hline $21-30$ & 45 & 42 & 93 & 32 & 71 & 42 & 93 \\
\hline $31-40$ & 30 & 27 & 90 & 19 & 63 & 30 & 100 \\
\hline $41-50$ & 13 & 10 & 77 & 11 & 85 & 13 & 100 \\
\hline $50+$ & 10 & 10 & 100 & 8 & 80 & 9 & 90 \\
\hline n.a. & 1 & 1 & 100 & 1 & 100 & 1 & 100 \\
\hline \multicolumn{8}{|c|}{ CGM/FGM use } \\
\hline yes & 117 & 104 & 89 & 96 & 82 & 112 & 96 \\
\hline no & 75 & 73 & 97 & 43 & 57 & 74 & 99 \\
\hline \multicolumn{8}{|c|}{ Insulin pump user } \\
\hline yes & 121 & 110 & 91 & 89 & 74 & 117 & 97 \\
\hline no & 71 & 67 & 94 & 50 & 70 & 69 & 97 \\
\hline \multicolumn{8}{|c|}{ Daily BG testing frequency } \\
\hline $1-2$ & 7 & 7 & 100 & 3 & 43 & 7 & 100 \\
\hline $3-4$ & 25 & 24 & 96 & 13 & 52 & 25 & 100 \\
\hline $5-7$ & 50 & 49 & 98 & 33 & 66 & 50 & 100 \\
\hline $8-12$ & 48 & 46 & 96 & 38 & 79 & 45 & 94 \\
\hline $13-17$ & 15 & 11 & 73 & 13 & 87 & 15 & 100 \\
\hline $18+$ & 46 & 39 & 85 & 38 & 83 & 43 & 93 \\
\hline n.a. & 1 & 1 & 100 & 1 & 100 & 1 & 100 \\
\hline \multicolumn{8}{|c|}{ Boluses/shots per day } \\
\hline $1-3$ & 14 & 14 & 100 & 6 & 43 & 14 & 100 \\
\hline $4-5$ & 99 & 94 & 95 & 68 & 69 & 97 & 98 \\
\hline $6-8$ & 53 & 45 & 85 & 45 & 85 & 51 & 96 \\
\hline $9-11$ & 21 & 19 & 90 & 16 & 76 & 19 & 90 \\
\hline $12+$ & 2 & 2 & 100 & 2 & 100 & 2 & 100 \\
\hline n.a. & 3 & 3 & 100 & 2 & 67 & 3 & 100 \\
\hline
\end{tabular}

Table 2 Survey results showing behaviors by demographic

Age in years

We found consistent and prevalent habitual and sensemaking behaviors across all age groups, with no indicators that any of the three categories are related to participant age.

\section{Gender}

We found no significant correlations related to gender.

\section{Time since diagnosis in years}

We found no statistical significance in correlation, although there might be a slight upward trend in FCR within the first 10 years after diagnosis.

\section{CGM/FGM use}

Non-users might have a small tendency towards more habitual responses as opposed to users. CGM users appear to have an increase in FCR classification as opposed to nonusers ( $82 \%$ vs. $57 \%$ ), a Fisher Exact Test showed a p $<0.01$, demonstrating significance in this category.

\section{Insulin pump use}

We do not see evidence for correlation between method of insulin delivery and reasoning modes.

\section{Daily BG testing frequency}

Frequency of daily testing appears to be associated with an increased tendency towards FCR categorization, as shown by bar graph progression in Fig. 2. Sensemaking appears relatively consistent among all groups. Habitual thinking might decrease slightly with increased testing frequency, though this might be due to the small sample in the '13-17' $(\mathrm{n}=15)$ group. Means were determined for each demographic, which were then used to perform a Kendall's Tau test, performed with the SciPy Stats package in Jupyter Notebook. The increase in FCR behavior was found to have a small but significant correlation with daily testing frequency (Tau 0.20, p < 0.001).

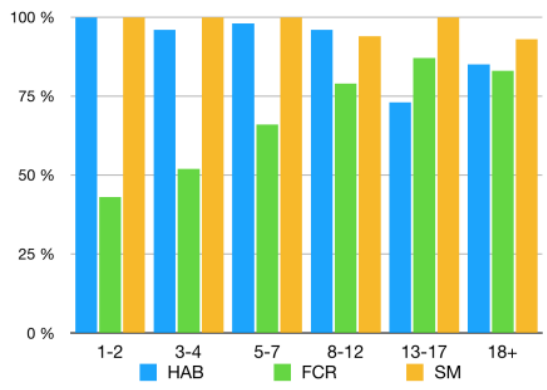

Figure 2 Daily testing freq. vs. $\% \mathrm{w} /$ Cognitive mode

\section{Boluses/shots per day}

While Sensemaking and Habitual modes appear relatively stable, there appears to be a small correlative relationship between greater frequencies of insulin injection and FCR mode. The same procedure was followed as in the previous paragraph, with indications of a small but significant correlation between boluses per day and FCR classification (Tau $0.19, \mathrm{p}<0.001)$.

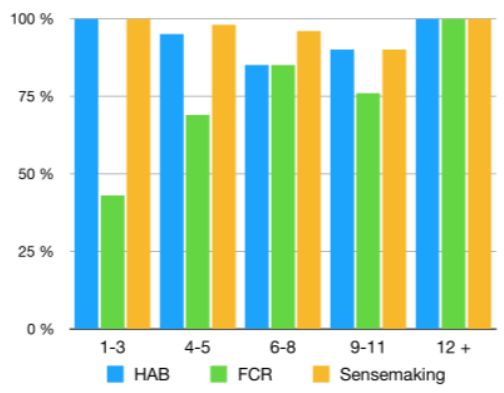

Figure 3 Bolus/day vs. \% Cognitive mode 


\section{Open-ended survey questions}

In the following sections, we will review result of the openended question portion of the survey.

\begin{tabular}{|c|c|c|c|c|c|c|}
\hline \multicolumn{3}{|c|}{ Habitual } & \multicolumn{2}{|c|}{ FCR } & \multicolumn{2}{|c|}{ Sensemaking } \\
\hline $\mathrm{n}=$ & $\mathrm{n}=$ & $\%$ & $\mathrm{n}=$ & $\%$ & $\mathrm{n}=$ & $\%$ \\
\hline \multicolumn{7}{|c|}{ Qo7. Normal BG/ response } \\
\hline 189 & 73 & 39 & 116 & 61 & 0 & o० \\
\hline \multicolumn{7}{|c|}{ Qo8. Recall surprising BG event } \\
\hline 166 & 23 & 14 & 6 & 04 & 137 & 83 \\
\hline \multicolumn{7}{|c|}{ Qo8b.Treatment procedure } \\
\hline 167 & 86 & 51 & 22 & 13 & 59 & 35 \\
\hline \multicolumn{7}{|c|}{ Qog. Out of range w. obvious cause } \\
\hline 178 & 17 & 10 & 12 & 07 & 149 & 84 \\
\hline \multicolumn{7}{|c|}{ Qogb. Treatment procedure } \\
\hline 177 & 132 & 75 & 21 & 12 & 24 & 14 \\
\hline \multicolumn{7}{|c|}{ Q10. Recall out of range event w/ novel situation } \\
\hline 143 & 2 & 01 & 3 & 02 & 138 & 97 \\
\hline \multicolumn{7}{|c|}{ Q1ob. Treatment procedure } \\
\hline 129 & 67 & 52 & 24 & 19 & 38 & 29 \\
\hline \multicolumn{7}{|c|}{ Q11. Change in diabetes management since diagnosis } \\
\hline 28 & 11 & 39 & 7 & 25 & 10 & 36 \\
\hline \multicolumn{7}{|c|}{ Q12. If CGM user, effect on management } \\
\hline 39 & 1 & 03 & 29 & 74 & 9 & 23 \\
\hline \multicolumn{7}{|c|}{ Q13. Current BG, report factors } \\
\hline 142 & 28 & 20 & 46 & 32 & 68 & 48 \\
\hline \multicolumn{7}{|c|}{ Q14. Management process for meal w/ current BG } \\
\hline 164 & 85 & 52 & 79 & 48 & 0 & 0 \\
\hline
\end{tabular}

Table 3 Counts of responses to open ended survey questions

Q07. Describe factors when deciding on insulin dosage under normal BG conditions

These responses appear to lend support to the [15] model, as without a 'gap' to initiate effortful thinking, we do not see the effortful sensemaking process. This question on routine management practices produced only habitual $(39 \%)$ and FCR $(61 \%)$ responses. Common examples of habitual responses were simple algorithms, such as fixed insulin to carb ratios. In contrast FCR was evidenced by answers by hard to quantify contextual situations such as P141 "Carbs, exercise, stress, illness." Also common were references to hypothetical events such as future exercise.

Q08. Describe situation w. serious and surprising blood glucose value

In contrast to the $Q 07$, this question was formulated to elicit a 'gap' situation response, which was reflected in $83 \%$ of responses being classified as sensemaking. P030 recounts, "I learned the hard way as a teenager the effects alcohol can have on your blood sugar. I took my normal bolus the next morning with breakfast and went back to sleep. Next thing I remember is waking up very confused, with a very painful mouth and body. "In this example, we see the learning process and formation of models related to alcohol consumption. Or P060 who stated, "I guessed wrong about carbs in a meal and over-bolused." Thereby showing awareness of a specific cause and effect relation. However, the second part of this question on treatment procedure resulted in substantial habitual responses (51\%). For example, P141 recounted treating unexpected hypoglycemia with " $15 \mathrm{~g}$ fast carbs then slow carbs like biscuits or bread." Thereby demonstrating the initiation of a script of behav- iors. Sensemaking was also common in $Q 08 b$ (35\%), typically the establishment of a new habitual model, for example P154 noted, "It made me consider potential options for keeping items by my bed to test or treat lows instead and keeping my phone close too in case I needed to call for help."

Q09. Describe out of range event w. obvious cause

We hypothesized that indication of an obvious cause would reduce sensemaking, in relation to the 'surprising event' of $Q 08$, however this was not reflected in our totals, which produced almost identical proportions to Q08. However, this discrepancy was reconciled in the second part of the question on treatment procedure, where we see substantially more habitual $(75 \%)$ responses, and a resultant drop in sensemaking behaviors, such as new model formation.

Q10. Recall out of range event w/ novel situation

This question was designed to evoke a specific 'gap' situation, an unwanted BG value in a novel situation. This led to $97 \%$ of participants responding with a sensemaking response. The second part of the question had similar response patterns as $Q 08 b$, with a majority (52\%) of habitual responses.

Q11. Change in diabetes management since diagnosis

Due to the small number of categorizable responses, this question will not be further discussed.

Q12. If CGM user, effect on management

While $Q 12$ also had only a small number of responses that could be categorized, $74 \%$ of responses were categorized as FCR, further supporting the link between CGM use and FCR in diabetes management.

Q13. Current BG, report factors

Asking participants to analyze their current BG produced $48 \%$ in the sensemaking mode, followed by $32 \%$ in the FCR mode. Habitual responses lagged at $20 \%$.

Q14. Management process for meal w/ current BG

This question prompted relatively even habitual and FCR responses (52\% vs. 48\%). Habitual responses often related the use of simple procedures. For example P205 answers, "I would only dose for the carbs I was about to eat. 1 unit for every 15 carbs." While FCR responses often show fluid balancing of multiple models and emotional factors, such as P199 noting "I would eat low carb if I have a high reading but as I'm at work I only have soup for lunch which is $18 \mathrm{~g}$ of carb so have to eat that, I know as I'm high it's not good as I would prefer to be in range before eating, but as I am hungry I need to eat, this is where I really get frustrated with T1, I would therefore take an extra $20 \%$ dose on insulin to bring me down, as when running above 12 I also become more insulin resistant."

\section{Discussion}

In the following sections, we will first consider the general findings regarding the proposed expanded cognitive framework for diabetes self-management: habitual, FCR, and sensemaking modes. Secondly, we will apply these three modes to discuss existing diabetes related UI's. And finally, 
we will discuss how the theoretical and practical outcomes of these study results could be applied to the design of diabetes related UI's.

\section{Habitual}

Habitual modes are a means of low-cognitive demand reaction, particularly important for treating out of range situations with obvious causes. We allow that treatment algorithms can be relatively complex, such as multi-factor calculation of insulin dosages, given that this remains context independent. As P136 notes about calculating insulin dosages, "I did in my head for years so the formula is burned into my brain." This mode is crucial for quick responses, especially as out of range conditions were frequently cited as causing cognitive impairment. Such as P064 describing a particularly bad hypoglycemic episode, "I was disoriented, sweaty, dizzy, couldn't see straight. I was able to test my blood sugar and it was 17. At that point, my only thought that I could muster was to eat whatever I could find to try to keep conscious." References to this mode decreased substantially in $Q 08 b$ and $Q 10 b$, where the novelty of the situation doubled the incidence of sensemaking behaviors. We concur with [15] that this mode is largely dependent on ready access to models incorporated through the sensemaking process, and that the low cognitive demands appear consistent with Type 1 thinking.

\section{Sensemaking}

In instances $(Q 08, Q 09, Q 10)$ where participants were asked to recall surprising out of normal range situations, sensemaking was a dominant mode. We concur with the [15] model that sensemaking remains an essential activity for building models, and we saw extensive evidence where participants recalled a specific situation that led to new habit formation or critical lesson. For example, P049 who described having a hypoglycemic episode on a plane, and not getting up to get glucose from the overhead bin because of an activated fasten seat belt sign. "I played by the normal rules, though I shouldn't have. I should have gotten up at the moment I realized I was getting into trouble." We see evidence that sensemaking can be seen as the foundation for both habitual and FCR modes. In its effortful ability to overcome assumptions, biases, or habitual behaviors, it is consistent with Type 2 thinking.

\section{FCR}

While FCR thinking is less prevalent than habitual and sensemaking modes, it appears common throughout all demographics, independent of gender or age. It appears associated with CGM use, frequent BG measurements, and frequent bolus injections, and possibly a slight increase within the first years after T1D diagnosis. These correlations are at least partially, by definition, due to characteristics such as 'frequent course correction' and 'watching of trends' being strongly enabled by CGM technologies and increased insulin application. It also possible that the building of the flexible models used in FCR are enabled by the frequent checking and increased interactions supported by CGM and potentially other technologies which enable convenient data access. FCR appears most commonly in response to "normal situations' (Q07 61\%), relating the factors of 'current $\mathrm{BG}^{\prime}(Q 13,32 \%)$, and for 'projected management process for a hypothetical meal $(Q 14,48 \%)$. It is neither a retrospective tool, $(Q 08,4 \%, Q 09,7 \%, Q 10,2 \%)$, nor a prevalent treatment mode for surprising $(Q 08 b, 13 \%)$ nor for out of range events $(Q 09 b, 12 \%, Q 10 b, 19 \%)$. FCR does not appear to be a replacement for the habitual and sensemaking modes, nor the correct method for all situations, especially such as treatment for excessive hyperglycemia, where the automatic response of habitual mode might be preferable. FCR might also not be ideal for unexplained circumstances, where the desire to avoid such situations in the future point towards sensemaking and formation of new preventive models. As FCR appears to share some traits of each of the two other principal modes, it might be viewed as existing somewhere in a spectrum between the two other modes. At the same time, leaving aside any claims about dual or tri-process cognitive models, FCR appears at the least to characterize distinctive user behaviors with apparent implications for UI design, as discussed below.

\section{Current Ul's}

In the following section, we will consider two commonly used UI's and consider how their elements might relate to the discussed theories.

\section{Diabetes Logging App Statistics UI's}

The diabetes logging app genre, previously discussed in the introduction/supporting technologies section is a common approach for situated diabetes self-management. Fig. 4 shows an example of such an app. This UI displays statistics that could help guide management through trend discovery. Using our coding scheme, we would classify this UI as requiring 'effort' to apply to management decisions, and that its utility would be for 'Reflective or Hypothetical thinking'. Therefore, this UI could be classified primarily as a sensemaking tool, and be used primarily for 'gap' discovery. Such gaps could be useful for drawing attention to the need to question existing habitual behaviors, or undergo hypothesis testing to form new models. Such an interface would have limited benefits for supporting habitual modes, or FCR modes, and given the lack of sensemaking in evidence from $Q 14$ (management process for current meal), would not typically be of use for individual treatment decisions. As such, it could be seen primarily as a tool for intermittent use. 


\section{CGM/FGM UI}

Figure 5 shows the UI for the Abbott Libre Flash Glucose Meter. There are four primary design elements: current time, current $\mathrm{BG}$, arrow showing direction and rate of change, and a graph showing the previous 8 hours of measurements. The time function could be a component of all three reasoning modes, dependent on context. For example, time checking can be habitual behavior. It can be useful for helping to establish context for FCR, or can be used as a sensemaking tool, for specific and determined problem solving. The BG value is largely a habitual tool, with reflexive interpretation of 'too high', 'too low' or 'about right', although it is also critical in FCR navigation and course correction, and could be interpreted according to the requirements of a given context. It could also trigger sensemaking mode, when departing significantly from expectations. We classify the arrow as largely an FCR tool, as its implications are dependent on multiple contextual factors such as insulin on board, time of day, food already eaten, desired state, and relation to recent state. The graph as well could be used in all modes, dependent on context. Its use could be habitual, for example to assure the user that they are generally in range over a given period; it could be used as an FCR tool for assisting in navigation through time, by helping to show general tendencies. It could also be an 'effortful' sensemaking tool, especially in conjunction with additional recorded data, to help understand specific cause and effect relationships. In summary, according to our coding scheme, this UI incorporates aspects of all three modes, and can be flexibly applied according to situated user requirements.

\section{Implications for future adaptive UI design}

The following sections discuss potential practical applications of this research for future UI development as related to the three modes. Our research suggests that a binary model of cognition could be insufficient as a diabetes UI design framework, offering inadequate support for dynamic management techniques. An interface that is specifically geared towards habitual (Type 1) thinking is quick to use, but might not engage the user's cognitive abilities to challenge their biases. Conversely a sensemaking (Type 2) interface, such as a robust diabetes diary app, offers a means of overcoming such biases through effortful analysis of collected data; however, the high cognitive costs associated with insight extraction could be a barrier to frequent interaction. So, the designer of a diabetes support systems using only a two-aspect model faces a challenging question: how can UI's support interactions with complex data in a manner that is of low enough cognitive demand to allow for frequent usage while still encouraging non-reflexive mindful engagement? The following section attempts to address this concern, the implications of an expanded model, and its application to design.

\section{Habitual: design implications}

The habitual mode appears most relevant for addressing out of range events with understandable causes. As these situa-

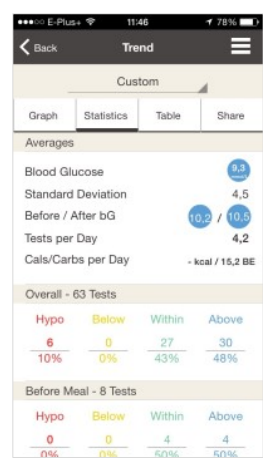

Figure 4 Diabetes Smartphone App

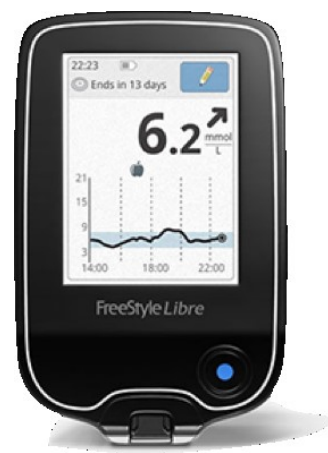

Figure 5 FGM tions can be cognitively and affectively stressful, interfaces should support and trigger familiar and easy to understand treatment steps. Wherever possible, such interfaces should attempt to help the user to integrate and link such behaviors into already existing behaviors, and thereby establish actions that become independent of interface interactions [22].

\section{FCR: design implications}

As FCR relies on the flexible use of multiple models, a supporting interface should help to trigger appropriate models at contextually relevant times and suggest other factors that might be relevant to support active engagement with diabetes management. As $F C R$ is a largely an in-themoment technique, graphs, charts and other cognitively demanding and primarily retrospective interface elements should be de-emphasized in favor of easy to understand elements such as short text, and icons where appropriate. Short animations might also be relevant. Ways to determine the appropriate personally relevant model triggers must be further researched, as well as means of determining personally relevant context for activation.

\section{Sensemaking: design implications}

Our study suggests that sensemaking is a dominant behavior when encountering out-of-range or novel situations. Traditional UI elements such as graphs and charts might be appropriate in such contexts to help users find specific correlations, or to help illustrate relevant insights. The emphasis should be on helping users to hypothesis test and construct new models, which can subsequently be used for less cognitively demanding habitual and FCR modes. As sensemaking is effortful, it is not for continual application; therefore, context awareness or some means of cognitive load sensing [20] might be used to determine when the user is amenable to such in depth engagement.

\section{Ethical considerations}

As interfaces for supporting medical practices seek to impact behaviors, their implementation has bio-ethical implications to principles such as beneficence, non-maleficence, justice, and autonomy [4]. Here we consider ethical concerns for each of the 3 cognitive models. 
As habitual behaviors can be critical for rapid responses, it is important that interfaces neither inhibit these reactions, nor interfere with successful strategies. This could argue against sustained UI engagement as a successful metric in such circumstances and point towards the importance of helping to support independent and intrinsically motivated behaviors. For example, if a user becomes dependent on their smartwatch to remind them to bring glucose when exercising, they might forget if their watch battery is empty. Such concerns could argue that ideally such critical behaviors should be technology independent when possible. As these behaviors are largely reflexive, respecting autonomy requires that persuasive and habit-forming systems are respectful of the personal preferences of the user, and have some means of assuring that the individual has made an informed decision.

As FCR depends on the access and adjustment of multiple self-care models, it is important that beneficial models are accessed, and adjusted in an appropriate manner. Ethical UI's might help this process by helping users to record personally relevant and desired self-care models, and then triggering these models in a contextually appropriate manner. Care must be taken that the user maintains the ability to choose what are appropriate suggestions without inappropriately forcing users into sensemaking mode, as this could inhibit frequent engagement.

As sensemaking is essential for forming self-care models, UI's should assist with informed investigation of beneficial models, while offering some means of warning about the formation of harmful models. Given that rapid access of such models is essential for the other modes, UIs should help to support integration of such models into practice. Care should be taken that users are educated into being able to engage in such practices independent of technology.

\section{CONCLUSIONS}

In this paper, we have shown evidence that many people with diabetes can make decisions with their complex data in a manner that does not easily conform with established cognitive models associated with habitual (Type 1 analogous), or sensemaking (Type 2 analogous) thinking. The former is largely reflexive, and therefore limited in its ability to exhibit variously: contextual consideration of complex multivariate data; hypothetical thinking as to potential events or outcomes; and awareness of highly variable situations. By contrast, the latter requires substantial cognitive effort, hence is ill suited to continual repeated application. While FCR is not necessarily a challenge to the fundamentals of two minds thinking, the behavior that we have evidenced does not readily match either. It appears conscious and engaged with complex scenarios, but there is not necessarily identification of a ' $g a p$ ' situation, nor is there inherent discarding or formation of new models. UI designs based solely on a two minds theory have to choose between two extremes, thereby offering no clear guidelines for frequent engagement that retains critical and hypothetical thinking. The inclusion of FCR could allow for such a state. While earlier work [23] appears relevant, we are not aware of integration into current diabetes UI design. Such an extension could help to provide a useful framework to analyze increasingly adopted tools such as CGM's, and help suggest new designs. FCR does not invalidate the other modes, and we observed no cases where participants displayed FCR without displaying the other modes as well. FCR could be viewed as a workaround, to conserve cognitive resources, using models built up through sensemaking and experience. Interestingly, it appears to be supported and fostered by emerging ubiquitous technologies, such as CGMs. FCR, while not as prevalent as habitual and sensemaking, was found to be common among all demographics within our survey population. We suggest that all three modes are important tools for diabetes self-management, and greater care should be taken that UIs for supporting self-management support these different modes within appropriate contexts.

Finally, we recognize that there is a significant body of research supporting dual-process theories, and it would be premature to challenge the perceived wisdom. However, there are more modest ways of viewing FCR mode as a useful cognitive workaround or adaption that allows individuals to accomplish domain specific requirements within the limitations of dual-process thinking. We have presented evidence that users appear to have found a way to integrate essential aspect of two recognized cognitive modes in order to interact with complex and hard to calculate data streams. This appears to allow them to weigh and make predictions on likely outcomes, and use those insights to make vital decisions, all in a fluid and frequent manner. We propose that support of such mental processes through medical UIs merits further exploration. We suggest that possible relevance to other domains would also bear investigation.

\section{LIMITATIONS}

This interaction research was conducted with a technology capable group and non-personal data which could have effects on results. The use of online recruitment could prejudice towards an engaged and knowledgeable sub-group, and therefore may not be broadly representative. Qualitative coding is always open to interpretation, especially regarding intent. As such this study is primarily exploratory, and not necessarily indicative of broader occurrences of these behaviors, nor how they may occur in 'in-the-wild' settings.

\section{FUTURE WORK}

The proposed theory should be taken as a starting point, and has not been validated by quantitative measurement of cognitive demands. A useful extension of this work would be to test the FCR hypothesis by creating and user testing UIs that facilitate FCR thinking.

\section{ACKNOWLEDGEMENTS}

Thanks to the participants, the many whose critical comments helped improve this paper, and Google Scholar program, UK EPSRC grants EP/L021285/1, EP/P01013X/1 \& ERC grant 291652 for research funding. 


\section{REFERENCES}

1. Florencia Aguiree, Alex Brown, Nam Ho Cho, Gisela Dahlquist, Sheree Dodd, Trisha Dunning, Michael Hirst, Christopher Hwang, Dianna Magliano, and Chris Patterson. 2013. IDF Diabetes Atlas.

2. American Association of Diabetes Educators. 2009. AADE Guidelines for the Practice of Diabetes SelfManagement Education and Training (DSME/T). The Diabetes Educator 35, 3 Suppl: 85S-107S. https://doi.org/10.1177/0145721709352436

3. Eirik Arsand, Dag Helge Frøisland, Stein Olav Skrøvseth, Taridzo Chomutare, Naoe Tatara, Gunnar Hartvigsen, and James T. Tufano. 2012. Mobile health applications to assist patients with diabetes: lessons learned and design implications. Journal of diabetes science and technology 6, 5: 1197-1206. Retrieved February 11, 2015 from http://dst.sagepub.com/content/6/5/1197.short

4. Tom L. Beauchamp and James F. Childress. 2001. Principles of biomedical ethics. Oxford University Press, USA.

5. Katherine Blondon, MSc1 Predrag Klasnja, and M. H. A. Logan Kendall. 2013. Long-term engagement with health-management technology: a dynamic process in diabetes1. Katherine S Blondon: 37. Retrieved November 17, 2016 from https://digital.lib.washington.edu/researchworks/bitstre am/handle/1773/23784/Blondon_washington_0250E_1 1783.pdf? sequence $=1 \&$ isAllowed $=$ y\#page $=4 \overline{4}$

6. Jonathan St. B. T. Evans and Keith E. Stanovich. 2013. Dual-Process Theories of Higher Cognition: Advancing the Debate. Perspectives on Psychological Science 8, 3: 223-241. https://doi.org/10.1177/1745691612460685

7. Martha Mitchell Funnell and Robert M. Anderson. 2000. The Problem With Compliance in Diabetes. JAMA 284, 13: 1709. https://doi.org/10.1001/jama.284.13.1709-JMS1004-61

8. Eric B. Hekler, Predrag Klasnja, Jon E. Froehlich, and Matthew P. Buman. 2013. Mind the theoretical gap: interpreting, using, and developing behavioral theory in HCI research. In Proceedings of the SIGCHI Conference on Human Factors in Computing Systems, 33073316. Retrieved March 7, 2016 from http://dl.acm.org/citation.cfm?id=2466452

9. Eric B. Hekler, Susan Michie, Misha Pavel, Daniel E. Rivera, Linda M. Collins, Holly B. Jimison, Claire Garnett, Skye Parral, and Donna Spruijt-Metz. 2016. Advancing Models and Theories for Digital Behavior Change Interventions. American Journal of Preventive
Medicine 51, 5: 825-832.

https://doi.org/10.1016/j.amepre.2016.06.013

10. Stephen S. Intille. 2004. Ubiquitous computing technology for just-in-time motivation of behavior change. Stud Health Technol Inform 107, Pt 2: 1434-7.

11. Dmitri Katz, Nick Dalton, Simon Holland, Aisling O'Kane, and Blaine A. Price. 2017. Questioning the Reflection Paradigm for Diabetes Mobile Apps. In eHealth $360^{\circ}$ : International Summit on eHealth, Budapest, Hungary, June 14-16, 2016, Revised Selected Papers, Kostas Giokas, Laszlo Bokor and Frank Hopfgartner (eds.). Springer International Publishing, Cham, 315-326. Retrieved from http://dx.doi.org/10.1007/978-3-319-49655-9_39

12. Ian Li, Anind K. Dey, and Jodi Forlizzi. 2011. Understanding my data, myself: supporting self-reflection with ubicomp technologies. In Proceedings of the 13th international conference on Ubiquitous computing, 405-414. Retrieved April 14, 2015 from http://dl.acm.org/citation.cfm?id=2030166

13. James J. Lin, Lena Mamykina, Silvia Lindtner, Gregory Delajoux, and Henry B. Strub. 2006. Fish'n'Steps: Encouraging Physical Activity with an Interactive Computer Game.

14. Lena Mamykina, Elizabeth Mynatt, Patricia Davidson, and Daniel Greenblatt. 2008. MAHI: investigation of social scaffolding for reflective thinking in diabetes management. In Proceedings of the SIGCHI Conference on Human Factors in Computing Systems, 477486. Retrieved February 11, 2015 from http://dl.acm.org/citation.cfm?id=1357131

15. Lena Mamykina, Arlene M. Smaldone, and Suzanne R. Bakken. 2015. Adopting the sensemaking perspective for chronic disease self-management. Journal of biomedical informatics 56: 406-417.

16. Rob Meredith and David Arnott. 2003. On ethics and decision support systems development. In On Ethics and Decision Support Systems Development. PACIS 2003 Proceedings. Paper 106. Retrieved Nove 6, 2016 from https://works.bepress.com/david_arnott/1/download/

17. Susan Michie, Maartje M. van Stralen, and Robert West. 2011. The behaviour change wheel: a new method for characterising and designing behaviour change interventions. Implementation Science 6, 1: 42. Retrieved May 30, 2016 from http://www.

implementationscience.com/content/6/1/42/

18. Kellee M. Miller, Nicole C. Foster, Roy W. Beck, Richard M. Bergenstal, Stephanie N. DuBose, Linda A. DiMeglio, David M. Maahs, and William V. Tamborlane. 2015. Current State of Type 1 Diabetes Treatment in the U.S.: Updated Data From the T1D 
Exchange Clinic Registry. Diabetes Care 38, 6: 971978. https://doi.org/10.2337/dc15-0078

19. Veljko Pejovic and Mirco Musolesi. 2014. Anticipatory mobile computing for behaviour change interventions. 1025-1034.

https://doi.org/10.1145/2638728.2641284

20. Sohail Rafiqi, Chatchai Wangwiwattana, Jasmine Kim, Ephrem Fernandez, Suku Nair, and Eric C. Larson. 2015. PupilWare: towards pervasive cognitive load measurement using commodity devices. In Proceedings of the 8th ACM International Conference on PErvasive Technologies Related to Assistive Environments, 42. Retrieved March 21, 2017 from http://dl.acm.org/citation.cfm?id=2769506
21. Keith E. Stanovich. 2009. Distinguishing the reflective, algorithmic, and autonomous minds: Is it time for a triprocess theory. In two minds: Dual processes and beyond: 55-88. Retrieved January 13, 2017 from http://keithstanovich.com/Site/Research_on_Reasoning _files/Stanovich_Two_MInds.pdf

22. Katarzyna Stawarz, Anna L. Cox, and Ann Blandford. 2015. Beyond self-tracking and reminders: designing smartphone apps that support habit formation. In Proceedings of the 33rd Annual ACM Conference on Human Factors in Computing Systems, 2653-2662.

23. Alexandra L. Varga and Kai Hamburger. 2014. Beyond type 1 vs. type 2 processing: the tri-dimensional way. Frontiers in Psychology 5.

https://doi.org/10.3389/fpsyg.2014.00993 